\section{MS12-P15 Crystallization of heat shock protein essential for protein disaggregation}

Marta Orlikowska ${ }^{1}$, Krzysztof Liberek $^{2}$, Grzegorz Bujacz ${ }^{1}$

1. Institute of Technical Biochemistry, Lodz University of Technology, Poland

2. Department of Molecular and Cellular Biology, University of Gdańsk

email: martaorlikowska07@gmail.com

The process in which a newly synthesized polypeptide chain transforms itself into a perfectly folded protein depends both on the properties of the amino-acid sequence and on multiple contributing influences from the crowded cellular milieu. Uncovering the mechanism of protein folding and unfolding is one of the grand challenges of modern science. The three-dimensional arrangement of the polypeptide chain decide about the specific biological function of the protein in the cell. Only correctly folded proteins are fully functional, randomly arranged polypeptide chain doesn't have biological activity. The state of protein folding is controlled and regulated by the protein quality control system. The system is formed by chaperones involved in protein folding and the proteasomal degradation system. The proper functioning of the system is required because its dysfunction may lead to neurodegenerative diseases. The prion-related illnesses such as Creutzfeldt-Jakob disease, amyloid-related illnesses such as Alzheimer's disease as well as intracytoplasmic aggregation diseases such as Huntington's and Parkinson's disease those are neurodegenerative diseases whose pathogenesis is associated with protein aggregation of incorrectly folded proteins. Many chaperones are heat shock proteins. Their expression is increased when cells are exposed to elevated temperatures or other stress conditions. The project focuses on the protein Hsp104 which belongs to the Hsp100 family and the AAA+ superfamily. Hsp104 is important in the cell due to its ability to solubilize and refold proteins trapped in aggregates formed during heat stress [1]. It achieves this in cooperation with the Hsp70 chaperone system. The active form of the protein is a ring-shaped hexamer, which is thought to drive protein disaggregation by directly translocating substrates through its central channel. However, there is still no general consensus regarding the domain organization within the hexameric molecular machine. Substantial efforts have been made to elucidate the location of domain $\mathrm{M}$, but the results are contradictory $[2,3]$. We aim to learn the orientation of the unique $M$ domain by solving the crystal structure of Hsp104 using X-ray crystallography.

[1] Liberek, K. et al., EMBO J. 2008, 27, 328-335.

[2] Lee, S. et al. J. Struct. Bio., 2004, 146, 99-105.

[3] Wendler, P. et al., Cell. 2007, 28, 1366-1377.

This work was supported by Grant 2013/08/S/NZ1/00750 from National Science Center $(\mathrm{NCN})$

Keywords: crystallization, heat shock protein

\section{MS12-P16 Dimeric cyanobacterial 1-Cys}

\section{Prx6 is a moonlighting protein}

Uwe H. Sauer ${ }^{1}$, Yogesh Mishra ${ }^{2}$, Michael Hall ${ }^{1}$, Kwangho Nam ${ }^{1}$, Stefan Jansson ${ }^{3}$, Wolfgang P. Schröder ${ }^{1}$

1. Department of Chemistry, Umeå University, SE-901 87 Umeå, Sweden

2. Department of Botany, Centre of Advanced Study in Botany, Institute of Science, Banaras Hindu University, Varanasi, India-221005

3. Umeå Plant Science Centre, Department of Plant Physiology, Umeå University, SE-901 87 Umeå, Sweden

email: uwe.sauer@umu.se

Peroxiredoxins (Prxs) are vital regulators of intracellular reactive oxygen species (ROS) levels in most organisms. Their activity depends on one or two catalytic cysteine residues, but no metal, as for classical Prxs ${ }^{1}$.

Here, we report results for the dimeric 1-Cys Prx6 protein from Anabaena sp. $\left(\right.$ AnPrx6) ${ }^{2}$. By combining activity assays, X-ray crystallography, NMR, SAXS, mass-spectroscopy and MD simulations we have gained new insights into 1-Cys Prx action. In particular, active site asymmetry and consorted movements of key active site residues affect the activity. Furthermore, we show that the dimeric AnPrx6 is a moonlighting protein with peroxidase and molecular chaperone activity, without change of oligomeric state. The dual function might have contributed to the survival of cyanobacteria ${ }^{3}$ in the harsh environments on earth over billions of years.

\section{References:}

1. Wood ZA, et al. (2003) Trends Biochem Sci 28(1):32-40.

2. Mishra Y, et al. (2011) Acta Crystallogr F 67(10):1203-1206.

3. Dietz KJ (2011) Antioxid Redox Signal 15(4):1129-1159.

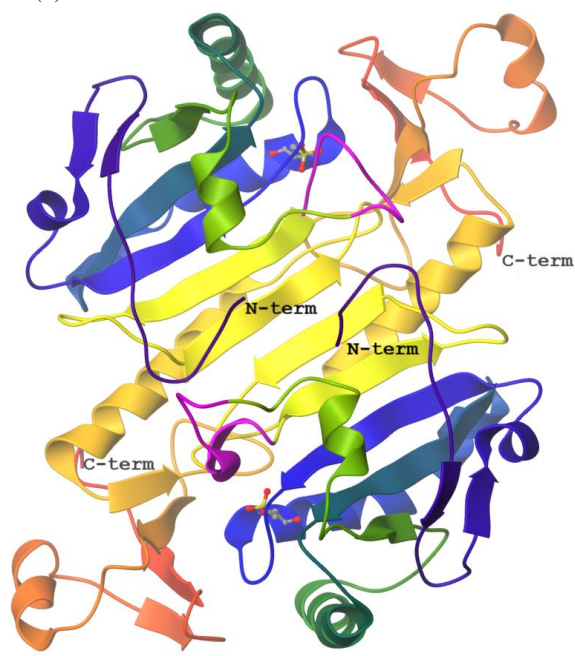

